\title{
Avaliação Clínica e Parasitológica de Cordeiros Suplementados com Subproduto de Descarte da Dieta da Larva da Mosca das Frutas $(C \text {. capitata })^{1}$
}

José Cláudio Barros Ferraz², Daniel Maia Nogueira ${ }^{3}$, Pedro Humberto Felix de Sousa ${ }^{4}$, Tadeu Vinhas Voltolini ${ }^{3}$, Tiago dos Santos Cruz ${ }^{5}$, Jadson Miranda Oliveira ${ }^{5}$, Emanuel Almeida Martinez

Resumo: Este trabalho objetivou avaliar os aspectos clínicos, monitorados pelo cartão de Famacha®, e parasitológicos, por meio da contagem no número de ovos por grama de fezes (OPG), de cordeiros mantidos em pastagem e suplementados com o subproduto de descarte da larva da mosca das frutas. Foram utilizados 40 ovinos, com idade inicial de dois a quatro meses. Os cordeiros foram mantidos em pastejo de capim-aruana (Panicum maximum cv. Aruana) durante oito horas diárias, e, posteriormente, suplementados com o feno do subproduto. A suplementação com o subproduto foi realizada nas quantidades de $0 \%$ (controle), 10\%, $20 \%$ e $30 \%$ com relação às exigências diárias de matéria seca estimada em 3,5\% de seu peso corporal. O delineamento experimental foi o inteiramente casualizado com quarto tratamentos e dez repetições. Os animais foram vermifugados de acordo com a coloração da conjuntiva comparada com o cartão Famacha ${ }^{\circledR}$ ou quando o OPG ficou acima de 1.500 ovos. A porcentagem de animais sadios, medianos, anêmicos e vermifugados não foram diferentes significativamente $(\mathrm{P}>0,05)$ entre os níveis de suplementação do subproduto. A porcentagem de animais vermifugados variou de 23,8 a 27,5\%. Não houve diferença significativa $(P>0,05)$ para o número de OPG entre os níveis de suplementação com o subproduto. Os níveis de suplementação do subproduto de descarte da dieta da larva da mosca das frutas não afetaram os parâmetros clínicos e parasitológicos dos ovinos.

Palavras-chave: anti-helmíntico, cartão Famacha ${ }^{\circledR}$, cordeiros, OPG, pastejo rotacionado, subproduto

\section{Clinical and Parasitological Evaluation of Lambs Supplemented with By-Product from Fruit fly Larvae (C. capitata)}

\footnotetext{
Abstract: This work aimed to evaluate the clinical, monitored by the Famacha ${ }^{\circledR}$ card, and parasitological aspects, monitored by the number of eggs per gram (EPG), of lambs maintained in pasture and supplemented with the by-product from fruit fly larvae. Forty lambs were used with initial age of two to four months. The lambs were maintained in capim-aruana grass (Panicum maximum cv. Aruana) for eight hours a day and supplemented with the hay of this by-product. The supplementation with the by-product was used in percentages of $0 \%$ (control), $10 \%, 20 \%$ and $30 \%$ in relationship the daily dry matter demands for $3.5 \%$ of body weight. It was used a random experimental design with four treatments and ten replicates. The animals were dewormed according to the color of conjunctiva compared with the Famacha ${ }^{\circledR}$ card or when EPG was above 1,500 eggs. The percentage of healthy, medium, anemic and dewormed animals were not significantly different $(\mathrm{P}>0.05)$ among the supplementation levels of the by- product. The percentage of dewormed animals varied from 23.8

${ }^{1}$ Projeto incentivado pela Biofábrica Moscamed Brasil

${ }^{2}$ Graduando em Agronomia - DTCS/UNEB. Bolsista da Biofábrica Moscamed Brasil. E-mail: claudioagronomia@gmail.com

${ }^{3}$ Pesquisador da Embrapa Semi-Árido em Petrolina-PE. E-mail: daniel@cpatsa.embrapa.br

${ }^{4}$ Professor do DTCS/UNEB em Juazeiro - BA. E-mail: rnbsouza@bol.com.br

${ }^{5}$ Discente do curso de Agronomia - DTCS/UNEB em Juazeiro-BA
} 
Rev. Cient. Prod. Anim., v.12, n.2, p.219-222, 2010

to $27.5 \%$. There was not significant difference $(\mathrm{P}>0.05)$ for the EPG among the supplementation levels of by-product. The supplementation levels of by-product from fruit fly larvae did not influenced the clinical and parasitological parameters of lambs.

Key words: anthelmintic, byproduct, EPG, Famacha ${ }^{\circledR}$ card, lambs, rotational grazing

\section{Introdução}

A contribuição das pastagens para a produção de carne no Brasilé extremamente elevada por ser a forma mais barata para a produção de volumosos, afinal, as condições climáticas favorecem o seu cultivo. Os subprodutos agroindustriais têm sido utilizados como suplemento ou até mesmo como fonte única de alimento na produção de ruminantes. No Brasil, o uso de subprodutos são estudados objetivando a redução do custo de alimentação dos animais, principalmente para os pequenos produtores rurais, e a possibilidade de diminuição do impacto ambiental dos resíduos agroindustriais.

Em pastagens irrigadas, os helmintos encontram condições favoráveis de temperatura e umidade para completar seu ciclo biológico e promover aumento da contaminação ambiental e, conseqüentemente, dos animais. Segundo Molento et al. (2004) o método de Famacha ${ }^{\circledR}$ permite a avaliação da mucosa ocular por meio dos diferentes tons de coloração, variando de vermelho-robusto até o quase branco, que está correlacionada como o grau de parasitismo por Heamonchus contortus, vermes hematófagos do abomaso dos pequenos ruminantes. Contudo, Nogueira et al. (2008) evidenciaram a fragilidade do cartão Famacha ${ }^{\circledR}$ para o diagnóstico de outras endoparasitoses que não as causadas por Haemonchus sp., verificando que esta metodologia não foi eficiente para reduzir a quantidade de ovos por gramas de fezes (OPG) a níveis inferiores a 1500. Portanto, o objetivo deste trabalho foi avaliar os aspectos clínicos, monitorados pelo cartão de Famacha®, e parasitológicos, através do controle do OPG, de cordeiros mantidos em pastagem e suplementados com o subproduto de descarte da larva da mosca das frutas (Ceratitis capitata).

\section{Material e Métodos}

O trabalho foi conduzido na área experimental do Departamento de Tecnologia e Ciências Sociais (DTCS), da Universidade do Estado da Bahia (UNEB), no município de Juazeiro-BA, no período de março a setembro de 2008. Foram utilizados 40 ovinos, sendo 20 Sem Raça Definida (SRD) e 20 Santa Inês, com idade inicial de dois a quatro meses, oriundos de estação de monta.

Estes animais foram divididos em quatro tratamentos, com 10 animais cada, sendo cinco da raça Santa Inês e cinco SRD, distribuídos uniformemente quanto ao peso e ao sexo. Os cordeiros foram mantidos em pastejo de capim-aruana (Panicum maximum cv. Aruana) durante oito horas diárias, e, posteriormente, foram suplementados com o feno do subproduto de descarte da dieta da larva da mosca das frutas. A suplementação foi realizada nas quantidades de $0 \%$ (controle), $10 \%, 20 \%$ e $30 \%$ com relação as exigências diárias de matéria seca estimada em 3,5\% de seu peso vivo.

As avaliações da coloração da mucosa ocular e contagens de OPG foram realizadas a cada 15 dias. O OPG foi realizado segundo a técnica de McMaster modificada por Gordon e Whitlock (Ueno e Gonçalves, 1998). A vermifugação foi realizada de acordo com a coloração da mucosa ocular utilizando- se como base o método Famacha ${ }^{\circledR}$ (Molento et al., 2004) e o OPG. Foram vermifugados os animais anêmicos (mucosa 
ocular de cor branca e rosado-pálido) e medianos (mucosa ocular rosada) ou quando o OPG ficou acima de 1500 ovos.

O delineamento experimental foi o inteiramente casualizado com quatro tratamentos e dez repetições. As porcentagens de animais sadios, medianos, anêmicos, vermifugados e porcentagens de larvas L3 identificadas na coprocultura foram comparadas entre os tratamentos pelo teste do Qui- quadrado. O número de OPG foi analisado pelo procedimento de ANOVA e as comparações ente médias foram realizadas pelo este de Tukey. Para todos os parâmetros foi adotado o nível de significância de 5\%.

\section{Resultados e Discussão}

A porcentagem de animais sadios, medianos, anêmicos e vermifugados não apresentaram grandes variações entre os níveis de suplementação com o subproduto $(\mathrm{P}>0,05)$. A porcentagem de animais vermifugados variou de 23,8 a $27,5 \%$, não havendo diferença significativa $(\mathrm{P}>0,05)$ entre os tratamentos (Tabela 1). Desse modo, o método de vermifugação utilizado promoveu uma redução de 72,5 a $76,2 \%$ no número de aplicações anti-helmínticas, considerando que $100 \%$ dos animais fossem vermifugados mensalmente.

Em condições experimentais semelhantes, utilizando o método Famacha ${ }^{\circledR}$, Nogueira et al. (2008) verificaram que a porcentagem média de animais sadios, medianos e anêmicos foi, respectivamente, de $44,2 \%, 42,1 \%$ e $13,65 \%$. Segundo os mesmos autores, embora alguns animais apresentassem uma mucosa ocular sadia, apresentaram elevada prevalência de Trichostrongylus spp. e, consequentemente, redução do desempenho produtivo.

Em todos os níveis de suplementação com o subproduto, o OPG manteve-se inferior a 1.500 ovos (Tabela 2), não havendo diferença significativa $(\mathrm{P}>0,05)$ entre os tratamentos. Segundo Ueno \& Gonçalves (1998), a contagem do OPG, tratandose de infecções mistas, na faixa de 500 a 800 ovos é classificada como uma infecção de grau leve, de 800 a 1.500 ovos, moderado, e acima de 1.500 ovos, pesado.

Em todas as coproculturas realizadas, foi identificada a prevalência de 78,4 a 89,1 \% de larvas de Trichostrongylus spp., seguido de 7,6 a 19,1\% de larvas de Haemonchus spp. (Tabela 2). Esses resultados corroboram com Nogueira et al. (2008), que observaram uma prevalência de 80 a 93,0\% de larvas de Trichostrongylus sp. em pastagens irrigadas no Vale do São Francisco. Todavia, esses resultados contrastam com outros estudos conduzidos em áreas de sequeiro do semi-árido Nordestino, que revelaram que mais de $80,0 \%$ da carga parasitária de caprinos e de ovinos é composta por Haemonchus contortus (Costa \& Vieira, 1984; Girão et al., 1992).

A eficácia do método Famacha ${ }^{\circledR}$ é maior quando a prevalência do $H$. contortus representar, pelo

Tabela 1 - Porcentagem de animais sadios, medianos, anêmicos, classificados de acordo com a coloração da mucosa ocular*, e de animais vermifugados, mantidos em pastagem de capim-aruana e suplementados com diferentes níveis do subproduto de descarte da dieta da larva da mosca das frutas

\begin{tabular}{lcccc}
\hline \multirow{2}{*}{ Animais } & \multicolumn{4}{c}{ Níveis de suplementação com subproduto ( $\pm \mathrm{dp})$} \\
\cline { 2 - 5 } & \multicolumn{1}{c}{$0 \%$} & \multicolumn{1}{c}{$10 \%$} & $20 \%$ & $30 \%$ \\
\hline Sadios (\%) & $53,3 \pm 0,13$ & $66,7 \pm 0,15$ & $560 \pm 0,17$ & $63,0 \pm 0,09$ \\
Medianos (\%) & $34,5 \pm 0,13$ & $23,4 \pm 0,12$ & $32,0 \pm 0,16$ & $26,0 \pm 0,13$ \\
Anêmicos (\%) & $12,2 \pm 0,07$ & $9,9 \pm 0,07$ & $12,0 \pm 0,04$ & $11,0 \pm 0,12$ \\
Vermifugados (\%) & $27,5 \pm 0,20$ & $25,0 \pm 0,13$ & $23,8 \pm 0,23$ & $26,3 \pm 0,24$ \\
\hline
\end{tabular}

* Sadios: vermelho-robusto (1) ou vermelho-rosado (2); Medianos: rosado (3); Anêmicos: rosado-pálido (4) ou branca (5). Não houve diferença significativa entre os tratamentos $(\mathrm{P}>0,05)$; Qui-quadrado calculado $<3,84$. 
Rev. Cient. Prod. Anim., v.12, n.2, p.219-222, 2010

Tabela 2 - Número médio de OPG e porcentagem de larvas infectantes L3 encontradas nas coproculturas de cordeiros mantidos em pastagem de capim-aruana e suplementados com diferentes níveis do subproduto de descarte da dieta da larva da mosca das frutas

\begin{tabular}{lcccc}
\hline \multirow{2}{*}{ Variáveis } & \multicolumn{4}{c}{ Níveis de suplementação com o subproduto } \\
\cline { 2 - 5 } & $0 \%$ & $10 \%$ & $20 \%$ & $30 \%$ \\
\hline Média de OPG (n) & 1269 & 1264 & 1268 & 1447 \\
Trichostrongylus spp. (\%) & 78,4 & 89,1 & 85,2 & 78,8 \\
Haemonchus spp. (\%) & 12,4 & 7,6 & 10,2 & 19,1 \\
Oesophagostomun spp. (\%) & 9,3 & 3,3 & 4,6 & 2,1 \\
\hline
\end{tabular}

Não houve diferença estatística entres os tratamentos ( $\mathrm{P}>0,05)$; Qui-quadrado calculado $<3,84$.

menos, $60 \%$ da carga parasitária, sendo o principal responsável causador da anemia nos animais (Chagas et al., 2007). Desta forma, em condições irrigadas, em virtude da menor prevalência de $H$. Contortus, o uso do Método Famacha ${ }^{\circledR}$ como parâmetro exclusivo para o monitoramento parasitológico pode não ser eficiente. Portanto, a avaliação clínica da mucosa ocular por meio do cartão Famacha ${ }^{\circledR}$ não deve ser usada como única base para monitoramento da verminose de ovinos em pastagens irrigadas. Segundo Molento (2004), o método Famacha ${ }^{\circledR}$ deve estar associado ao conjunto de técnicas de um sistema integrado de manejo parasitário.

A metodologia baseada na avaliação da mucosa ocular pelo método Famacha ${ }^{\circledR}$ associada ao controle do OPG foi capaz de manter o OPG com valores inferiores a 1.500 para os diferentes níveis de suplementação do subproduto de descarte da dieta da larva da mosca das frutas.

\section{Conclusão}

Os níveis de suplementação do subproduto de descarte da dieta da larva da mosca das frutas não afetaram os parâmetros clínicos e parasitológicos dos ovinos mantidos em pastagem de capim-aruana.

\section{Literatura Citada}

CHAGAS, A.C.S., OLIVEIRA, M.C.S., CARVAlHO, C.O., MOLENTO, M.B. Método Famacha ${ }^{\circledR}$ : um recurso para o controle da verminose em ovinos. Circular Técnica, 52. Embrapa Pecuária Sudeste, São Carlos-SP, 2007.

COSTA, C.A.F.; VIEIRA, L.S. Controle de nematódeos gastrintestinais de caprinos e ovinos do estado do Ceará. Comunicado Técnico, 13. 6p. Embrapa Caprinos, Sobral-CE, 1984.

GIRÃO, E.S.; MEDEIROS, L.P. GIRÃO, R.N. Ocorrência e distribuição estacional de helmintos gastrintestinais de caprinos no município de Teresina, Piauí. Ciência Rural, v.22, p.197-202, 1992.

MOLENTO, M. B.; TASCA, C.; GALlO, A.; FERREIRA, M.; BONONI, R.; STECCA, E. Método Famacha como parâmetro clinico individual de infecção por "Haemonchus contortus" em pequenos ruminantes. Ciência Rural, v. 34, n. 4, p. 1139-1145, jul/ago. 2004.

NOGUEIRA, D. M.; MISTURA, C.; VOLTOLINI, T. V.; TURCO, S. H. N.; ARAÚJO, G. G. L. de; LOPES, A. M. G.; SOUZA, T. C. Avaliação clínica, parasitológica de fezes e produtiva de cordeiros em pastagens de capimaruana irrigado e adubado com diferentes doses de nitrogênio. In.: $45^{\circ}$ Reunião Anual da Sociedade Brasileira de Zootecnia (SBZ), CD Rom. Lavras-MG, 2008.

UENO, H., GONÇALVES, P.C. Manual para diagnóstico de helmintoses de ruminantes. 4ed., Tokyo: Japan International Cooperation Ageny (JICA). 1998. 143p. 